ISSN 1112-9867

http://www.jfas.info

\title{
PORTABLE DEVICES FOR DELIVERING IMAGERY AND MODELLING INTERVENTIONS: EFFECTS ON NETBALL PLAYERS'ADHERENCE, SHOOTING PERFORMANCE, AND SELF-EFFICACY
}

\author{
T. K. A. Khan ${ }^{1, *}$, T. Morris ${ }^{2}$ and D. Marchant ${ }^{2}$ \\ ${ }^{1}$ Faculty of Sports Science and Coaching, Universiti Pendidikan Sultan Idris, Tg Malim, \\ Perak, Malaysia \\ ${ }^{2}$ ISEAL, College of Sport and Exercise Science, Victoria University, Australia.
}

Published online: 10 November 2017

\begin{abstract}
The main objective of this study was to investigate the effectiveness of portable devices (MP4) and a stationary device (DVD and fixed point stationary computer) in delivering imagery and modelling training among female netball players, examining the effect on imagery adherence, performance, self-efficacy, and the relative efficacy of presenting imagery instructions once at the start or before each of the video modelling examples. Fifteen participants were randomly assigned into each of the MP4 (initial and repeated instructions) and DVD conditions. Participants $(\mathrm{N}=45)$ completed a measure of self-efficacy for netball shooting and performed a netball-shooting test. Then, they practised imagery for seven days. Finally, they repeated all the measures. The results showed shooting performance of participants in the portable device conditions improved significantly $(\mathrm{p}<.05)$ compared to those in the stationary device condition, but there was no significant shooting performance difference between the two portable device conditions. These results indicate that MP4 was a useful portable device to enhance imagery with video modelling training adherence and to improve performance.
\end{abstract}

Author Correspondence, e-mail: thariq@fsskj.upsi.edu.my doi: http://dx.doi.org/10.4314/jfas.v9i6s.98 
Keywords: imagery, video-modelling, portable device, self-efficacy.

\section{INTRODUCTION}

Imagery training based on video modelling has become a preferred method of delivering imagery training compared to the traditional method using a written imagery script [1]. For example, imagery training combined with video modelling is practical, especially for individuals who have some difficulties practising imagery due to lack of experience in specific events (e.g., Olympics, Tour de France) or limited sports and imagery skills [2]. Therefore, practitioners have regularly used a combination of imagery and modelling techniques with athletes in training, the main reason being to maximise their athletes' potential [3]. Researchers found that the combination of imagery and video modelling training affected tennis players' closed skills performance [4] and was effective in facilitating both closed and open skills during the learning process [5]. Results of a study indicated that it is useful imagery practice to conduct imagery training in conditions similar to the practice and competition environments [6]. Exploring the PETTLEP imagery training model developed by Holmes \& Collins [7], researchers are using some elements of video modelling as has been suggested. For example, sport psychologists have suggested that PETTLEP imagery, using video modelling, enhanced strength performance [8]. Thus, imagery interventions based on video modelling and combined imagery and modelling techniques have been recommended to be a pragmatic method to enhance learning for various sports and learning circumstances [9] a modelling video is used to practice imagery, a device is needed to view the video footage. Today, inventions using high-technology devices have improved efficiency in many areas of daily life. For example, telecommunications and audio-visual devices, namely the mobile telephone, DVD players, laptop computer computers, and iPod Touch, have been equipped with multi-purpose functions, are cost effective, user friendly, and have high mobility. Thus, it seems likely that there is potential for using the new technological devices in the process of delivering imagery training that includes video modelling. Various devices have been used to deliver psychological skills training, particularly for imagery and modelling training $[2,10]$. Specifically, high technology devices have become popular in the delivery of imagery and modelling $[11,12]$. Some of these devices, such as biofeedback machines, MP4, neuroimaging analysis devices, computer editing software, and audio-video recorders or players, have not just 
assisted in the delivery of such psychological skills training, but have also increased the effectiveness of the psychological skills training [13, 14].

Busy athletes with competing time demands have not always given priority to video-based imagery training that involved substantial time commitment and effort to visit stationary locations [8]. The development of technology from VCR and DVD players attached to monitors, to laptop computers and recently to small hand-held devices such as MP4 players, tablet devices, and smart phones offers attractive alternatives. Several sport psychologists who have used video equipment intensively to facilitate individual target behaviour performance have proposed further exploration on the advantages in using highly mobile devices [15]. Moreover, the devices selected for use in sport settings must be portable enough to capture video footage of athletes' actions especially on the field. Recent trends in the development of technology have shown extensive innovations and the production of portable devices with audio-video recording and display functions. Morris, Spittle [16] recommended that researchers should pay more attention to investigating the potential of using portable video devices to deliver imagery interventions. For example, Rymal, Martini [17] reported that rookie athletes used a portable device, specifically the iPod, before a game, however, examination of the literature did not identify any research examining the relative efficacy of the strategy of employing portable devices in imagery and modelling training.

Previous research data indicated that there are some potential benefits of employing portable devices to deliver imagery and modelling training, therefore, the main aim of this study was to examine the effectiveness of portable devices (MP4) tin the delivery of imagery and modelling training among netball players compared to a stationary device procedure (DVD). To investigate whether use of a portable device affected adherence to the intervention compared to a stationary device, and whether the portable and stationary devices enhanced athletes' netball shooting performance and self-efficacy. A secondary aim was to examine whether different imagery instructions influenced the effectiveness of imagery training. The data from this study was expected to provide information and guidance for the use of portable devices to deliver imagery and modelling training. It was predicted that participants in both portable device conditions (initial and repeated instructions) would have higher levels of adherence in imagery and modelling training than those in the stationary device condition. We predicted that participants 
using the portable device would (i) adhere more to use of the imagery and modelling training, (ii) improve performance more, and (iii) report higher levels of self-efficacy compared to participants who used the stationary device procedure (control condition).

\section{METHOD}

\subsection{Participants}

We recruited 45 female netball players (mean age $=24.9$ years, $S D=5.60$ ) for this study. All had specific skills in shooting and experience playing either as a goal attack or goal shooter and competing in a local netball league. All participants are best described as active non-elite players with senior competition experience (mean $=4.18$ years, $S D=1.45)$.

\subsection{Measures}

\subsubsection{Imagery Ability}

The Sport Imagery Ability Measure (SIAM; Watt et al., 2004) is a 48-item self-report questionnaire that examines the experience of 60 seconds of imagery of each of four sport-related scenes on 12 sub-scales. The SIAM has internal consistency reliability ranging from good to very good with the alpha coefficient values from .66 (speed subscale) to .87 (gustatory subscale) and moderate to very good test-retest reliability correlations for specific subscales varying from .44 (speed) to .83 (gustatory).

\subsubsection{Imagery log}

Participants in the portable device conditions used the electronic notes application on the MP4 (iPod Touch) that automatically recorded the date and time of their imagery activities (electronic imagery $\log$ ). An imagery logbook was provided to participants in the control condition (DVD), which they were instructed to complete for every imagery session.

\subsubsection{Practice shooting performance}

Participants' shooting performance was evaluated through 45 shots from nine different positions, three angles (i.e., left and right $45^{\circ}$ angle from goal line, middle $90^{\circ}$ angle in front of netball posts) and three distances (i.e., 0.98, 1.96, and 2.94 metres). Shooting outcome was measured by accumulating the total score from 45 shots (percentage of successful shots) and the shooting accuracy scoring system replicated previous studies [19]. Three points were given for a 
successful "clean" shot, two points for a successful shot with the ball touching a part of the goal ring, and one point for an unsuccessful shot, but the ball touched the goal ring. Zero was scored when the ball completely misses the goal ring. The performance task had a scoring range from 0 (poorest performance: every shot misses the goal ring completely) to 135 (best possible performance: every shot is a clean goal). All the equipment and dimensions of the task in this study complied with international netball rules [20].

\subsubsection{Self-efficacy for netball shooting}

The self-efficacy measure was developed, using guidelines presented by [21], based on his original procedures for developing a task specific measure of self-efficacy, known as the microanalytic technique. Participants rated their confidence to perform at each of 8 levels of netball shooting from the lowest level of 10 out of 45 shots, increasing in 5-shot intervals to the highest level, that is, 45 out of 45 . Participants assessed their confidence to shoot at each level from 0 (certain cannot do at all) to 100 (certain can do). The final score was the sum of confidence ratings for all 8 levels of performance, divided by 8 , thus, resulting in a range from 0 (low self-efficacy) to 100 (high self-efficacy).

\subsection{Equipment and Specifications}

The audio instruction and video recording for the imagery training sessions were prepared using a JVC Everio Camcorder digital video camera (model GZ-HD300). The video footage was edited using the JVC Everio Media Browser software. The imagery audio instruction and video modelling were downloaded onto the portable device using iTunes software. An Apple iPod Touch was selected as the portable device (MP4) to deliver imagery and modelling training. The same imagery audio instructions and video modelling material were also downloaded onto DVD for delivery using the stationary device.

\subsection{Interventions}

The imagery instructions were developed to direct the participants' attention to the correct techniques for performing successful shots in netball. The video modelling employed an expert female netball player model [23], shooting with the correct techniques (Netball Australia high performance goal shooting notes). The netball shooting video model displayed $100 \%$ success. 
The shooting was performed from the same nine positions and angles as the experimental shooting test. The selection of model shooting examples and audio instructions were made with advice from two experienced netball coaches (Netball Australia, advance coaching accreditation). The imagery instructions and video modelling were downloaded onto the MP4 or onto a DVD. Participants were advised to use the portable devices or the DVD provided, depending on their assigned research condition as imagery training at least once a day.

\subsection{Procedure}

Voluntary participation was invited from among the netball shooter players participating in a local netball competition after ethics approval from the authors' institution. First, the SIAM was administered to screen for at least moderate levels (from 150 to 400) of self-reported imagery ability on the visual, kinaesthetic, vividness, and controllability subscales. This imagery ability screening procedure was used to ensure that all those who participated had the capability to benefit from the imagery training. Participants were randomly assigned to one of the three imagery conditions, with equal numbers of participants in the Initial Instructions Condition, the Repeated Instructions Condition, and the Control Condition.

The self-efficacy measure was administered to gain a baseline self-efficacy score for the shooting task (pre-test 1; Figure 1) before the shooting performance test was conducted. Participants completed the 45 test shots in blocks of five from each location, with 30 seconds rest between the shooting locations. Next, the self-efficacy measure was administered once more to obtain participants' level of self-efficacy after experiencing the shooting performance test (pre-test 2). The intervention was commenced immediately after the shooting performance and self-efficacy pre-test. Participants were provided with the portable devices and instructed to perform the imagery training at least once a day for the duration of seven days. Participants were also advised to record their imagery training activities using the electronic notes application on the portable devices. Participants in the C Condition were provided with a DVD that included the same imagery-training program content as the II Condition and the same video modelling. They were also instructed to use the DVD provided using a stationary computer and advised to record all their imagery training activities in the logbook provided. On Day 7, when the intervention finished, all participants repeated the self-efficacy measure (post-test 1) and then the shooting 
performance test. The final self-efficacy measure was administered immediately after the shooting performance test (post-test 2).

\section{Self-efficacy measure flow}

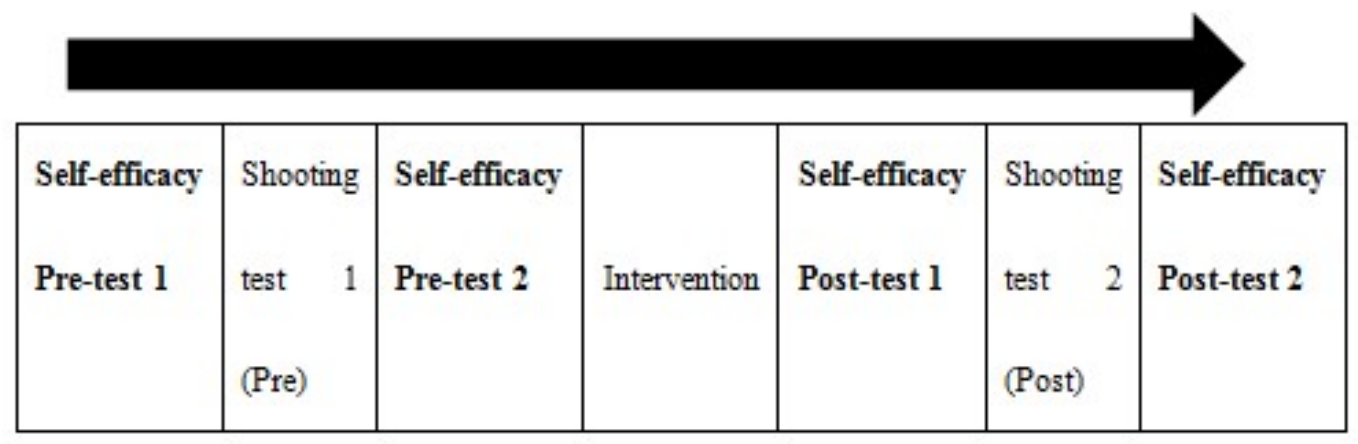

Fig.1. Self-efficacy measure flow chart

\section{RESULTS}

\subsection{Devices Frequency of Use}

MANOVA was employed to examine any significant differences between the two portable device conditions (II and RI) and the DVD stationary procedure (C) in terms of usage rate during the seven days of the imagery-training intervention between all three conditions. The means and SDs showed that the usage frequency of the portable device for the II Condition ( $M$ $=7.80, S D=0.94)$, the RI Condition $(M=7.73, S D=1.16)$, and the C Condition $(M=4.40, S D$ $=1.18$ ) indicated very similar usage for the two portable device conditions, each exceeding one session per day, whereas usage in the DVD condition was much lower. Using a Bonferroni adjusted alpha level of .017 , there was a significant difference on frequency of device usage between conditions $F(12,74)=45.51, p<.0005$, partial $\eta^{2}=.68$, with a very large effect size. The Tukey HSD post-hoc test results showed a significant difference between the II Condition (scored higher) and the C Condition $(p<.0005)$, and a significant difference between the RI Condition (scored higher) and the C Condition $(p<.0005)$. There was no significant difference between the II Condition (scored higher) and RI Condition $(p=1)$. Using a Bonferroni adjusted alpha level of .017 , there was a significant difference on frequency of device usage between conditions $F(12,74)=45.51, p<.0005$, partial $\eta 2=.68$, with a very large effect size. The Tukey HSD post-hoc test results showed a significant difference between the II Condition (scored 
higher) and the C Condition $(p<.0005)$, and a significant difference between the RI Condition (scored higher) and the $\mathrm{C}$ Condition $(p<.0005)$. There was no significant difference between the II Condition (scored higher) and RI Condition $(p=1)$.

\subsection{Netball Shooting Performance}

With a Bonferroni adjusted alpha level of .017, the MANOVA for netball shooting performance showed that there was a significant difference between the shooting outcome gain scores for conditions, $F(12,74)=4.419, p=<0.005$, partial $\eta^{2}=.17$. The Tukey HSD post-hoc test showed significant differences between the II Condition (scored higher) and C Condition, and between the RI Condition (scored higher) and C Condition on shooting outcome gain score $(p<0.05)$. Conversely, no significant difference was found between the II Condition (scored higher) and the RI Condition $(p=.99)$.

Using a Bonferroni adjusted alpha level of .017, MANOVA showed that there was a significant difference on shooting accuracy gain scores between conditions, $F(12,74)=4.42, p<0.005$, partial $\eta^{2}=.23$. Tukey HSD post hoc test results showed significant differences for the shooting accuracy gain score between the II Condition (scored higher) and C Condition $(p<0.005)$, and between the RI Condition (scored higher) and C Condition $(p<0.05)$. No significant difference was found between the II Condition and the RI Condition (scored higher; $p=.97$ ).

\subsection{Self-efficacy for Netball Shooting}

The MANOVA results on self-efficacy gain scores show no significant differences between any conditions in any of the four comparisons $F(12,74)=0.17, p=0.98$, partial $\eta^{2}=.001$, with a very small effect size. The analysis signifies that the use of the portable device and stationary device procedures had limited effects on participants' shooting self-efficacy and there were no differences between conditions.

\section{DISCUSSION}

Consistent with the study hypothesis the results shown that participants in the portable device conditions employed the device more frequently compared to the participants using the stationary device (DVD). Employing this MP4 portable method participants' netball shooting performances were significantly stronger than with the DVD and desktop computer (stationary 
method). The MP4 is suggested to be a promising technique to deliver imagery and modelling training as athletes' shooting performances improved significantly. Researchers have associated imagery and modelling training with athletes' performance improvement in various sports [e.g., $24,25,26]$. This performance improvement can also be related to the imagery and modelling training adherence factor, indicating that one reason why participants in the II and RI portable device conditions recorded greater performance improvement than the stationary device was because they employed their MP4 more regularly than participants in the C Condition used the DVD and computer approach. Finally, although participants in the II and RI Conditions recorded greater performance improvement, participants in the C Condition also showed some improvement. The overall performance improvement in the C Condition supports previous research findings stating that imagery delivered by more traditional modes does facilitate sport performance [27]. Moreover, the reason why the participants in the C Condition recorded relatively lower performance increment rate compared to the participants in the portable device condition could largely be due to the frequency of device usage and not due to the variation in the delivery method.

The two types of instruction were compared to examine whether regularly reminding participants of the procedure to use the MP4 for imagery and modelling training in the RI Condition would enhance imagery effectiveness compared to the initial instructions only in the II Condition. Analyses revealed that there was no significant difference between the two instructions conditions in terms of adherence or performance enhancement. Thus, both types of instruction had similar effects in delivering imagery training, leading to enhanced performance. This suggests that instructions at the beginning of an imagery training session are as effective as repeated instructions, so there is no need to repeat instructions, which takes up extra time and could, in some circumstances or for some individuals, be distracting.

Change in self-efficacy as a result of doing imagery training was also examined in this study. The statistical analysis indicated that none of the devices used in this study significantly affected participants' self-efficacy. This self-efficacy result contradicted what has been proposed by many researchers, which is that imagery practice normally effects athletes' self-efficacy [28]. Moritz, Feltz [29] noted that the level of correlation or correspondence between measures of self-efficacy and performance depends on the extent to which the 
measures tap into the same elements of the task, because of the task-specific nature of self-efficacy. They called this the degree of concordance between measures of self-efficacy and performance. It is possible that there was a low level of correlation between self-efficacy and performance in the present study because of low concordance. Examination of the measures employed, however, indicates that in the self-efficacy measure participants were asked to assess their confidence in performing netball shots from three angles and three distances. In the performance measure they actually performed shots from those same angles and distances. Thus, based on the definition of Moritz et al. concordance between the self-efficacy measure and the performance measure in this study was very high, so this is not a viable explanation of the poor relationship between self-efficacy and performance.

\section{ACKNOWLEDGEMENTS}

Researchers would like to thank the participants that involved in this study.

\section{REFERENCES}

[1] Seif-Barghi, T., et al., The effect of an ecological imagery program on soccer performance of elite players. Asian Journal of Sports Medicine, 2012. 3(2): p. 81.

[2] Weinberg, R., Does imagery work? Effects on performance and mental skills. Journal of Imagery Research in Sport and Physical Activity, 2008. 3(1): p. 1-21.

[3] Bull, S.J. and C.J. Shambrook, Soccer: The mind game2004: Crowood Press.

[4] Coelho, R.W., et al., Imagery intervention in open and closed tennis motor skill performance. Perceptual and Motor Skills, 2007. 105(2): p. 458-468.

[5] Weinberg, R.S. and D. Gould, Foundations of Sport and Exercise Psychology, 6E2014: Human Kinetics.

[6] Wakefield, C. and D. Smith, From strength to strength: A single-case design study of PETTLEP imagery frequency. The Sport Psychologist, 2011. 25(3): p. 305-320.

[7] Holmes, P.S. and D.J. Collins, The PETTLEP approach to motor imagery: A functional equivalence model for sport psychologists. Journal of Applied Sport Psychology, 2001. 13(1): p. $60-83$. 
[8].8. Wright, C.J. and D. Smith, The effect of PETTLEP imagery on strength performance. International Journal of Sport and Exercise Psychology, 2009. 7(1): p. 18-31.

[9] Ramsey, R., et al., Examining the emotion aspect of PETTLEP-based imagery with penalty taking in soccer. Journal of Sport Behavior, 2010. 33(3): p. 295.

[10] Shearer, D., et al., The effects of a video-aided imagery intervention upon collective efficacy in an international paralympic wheelchair basketball team. Journal of Imagery Research in Sport and Physical Activity, 2009. 4(1).

[11]Bell, R.J., C.H. Skinner, and M.K. Halbrook, Solution-focused guided imagery as an intervention for golfers with the yips. Journal of Imagery Research in Sport and Physical Activity, 2011. 6(1).

[12]Ramirez, F.R., PETTLEP imagery and video-observation: a motivation case study of four badminton players. Polish Journal of Sport \& Tourism, 2010. 17(4).

[13]Davis, P.A. and W.E. Sime, Toward a psychophysiology of performance: Sport psychology principles dealing with anxiety. International Journal of Stress Management, 2005. 12(4): p. 363

[14]Guillot, A., et al., Motor imagery and motor performance: evidence from the sport science literature. The Neurophysiological Foundations of Mental and Motor Imagery, 2010: p. $215-226$.

[15]Hars, M. and C. Calmels, Observation of elite gymnastic performance: Processes and perceived functions of observation. Psychology of Sport and Exercise, 2007. 8(3): p. 337-354. [16]Morris, T., M. Spittle, and A.P. Watt, Imagery in sport2005: Human Kinetics.

[17]Rymal, A.M., R. Martini, and D.M. Ste-Marie, Self-regulatory processes employed during self-modeling: A qualitative analysis. Sport Psychologist, 2010. 24(1): p. 1-15.

[18]Watt, A.P., T. Morris, and M.B. Andersen, Issues in the development of a measure of imagery ability in sport. Journal of Mental Imagery, 2004. 28(3\&4): p. 149-180.

[19]Pates, J., et al., Effects of asynchronous music on flow states and shooting performance among netball players. Psychology of Sport and Exercise, 2003. 4: p. 415-427.

[20]Shakespear, W. and M. Caldow, Netball 2nd Edition: Steps to Success2009: Human Kinetics. 
[21]Bandura, A., Guide for constructing self-efficacy scales. Self-efficacy Beliefs of Adolescents, 2006. 5(307-337).

[22]Weir, M. and A.W.S. Watson, A twelve month study of sports injuries in one Irish school. Irish Journal of Medical Science, 1996. 165(3): p. 165-169.

[23]Ericsson, K.A. and J. Smith, Toward a general theory of expertise: Prospects and limits 1991: Cambridge University Press.

[24]Bernier, M. and J.F. Fournier, Functions of mental imagery in expert golfers. Psychology of Sport and Exercise, 2010. 11: p. 444-452.

[25]Fournier, J.F., S. Deremaux, and M. Bernier, Content, characteristics and function of mental images. Psychology of Sport and Exercise, 2008. 9: p. 734-748.

[26]Seif-Barghi, T., et al., The effect of an imagery ecological imagery program on soccer performance of elite players. Asian Journal of Sports Medicine, 2012. 3(2): p. 81-89.

[27]Cumming, J. and R. Ramsey, Imagery interventions in sport, in Advances in applied sport psychology: A review, S.D. Mellalieu and S. Hanton, Editors. 2008, Routledge: Hoboken. [28]Feltz, D.L., S.E. Short, and P.J. Sullivan, Self-efficacy in Sport. 2008: Human Kinetics. [29]Moritz, S.E., et al., The relation of self-efficacy measures to sport performance: A meta-analytic review. Research Quarterly for Exercise and Sport, 2000. 71(3): p. 280-294.

\section{How to cite this article:}

Azizuddin Khan T. K., Morris T., and Marchant D. Portable Devices for Delivering Imagery and Modelling Interventions: Effects on Netball Players' Adherence, Shooting Performance, and Self-Efficacy. J. Fundam. Appl. Sci., 2017, 9(6S), 1334-1345. 